\title{
BIOTIPOS KILLER DEL GENERO Candida AISLADOS DE RATONES
}

\author{
(Killer hiotypes of the gentes Candida isolated from mice.)
}

\author{
Hortensia M. Magaro R.'\& María E. A . Tosello G.' \\ Dplo de Microbiologíal 'Arca Palrasiologia,' CEREMIC. \\ Fac. de Cs. Bioq. y Farm. UNR. Suipacha 531 . Rosario 2000,
}

Palabras dave: Biolipos. toxinas killer Cam halat, moncs Key words: Killer loxins biolypes. Candida. mice

\section{RESUMEN}

Se terermini lo potencialiad hiller te di-

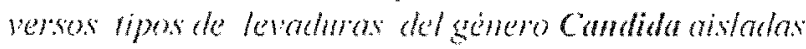

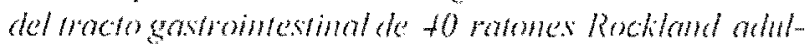
tos sometides a wha diela hipershoida, compuesta de

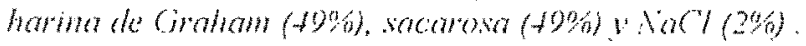

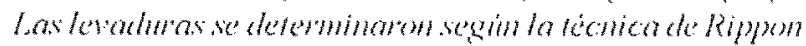

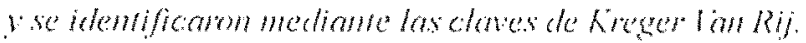

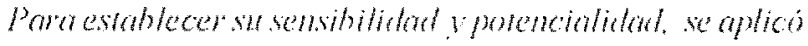

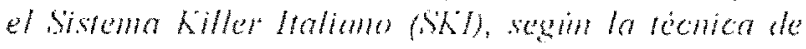
polonellieral.

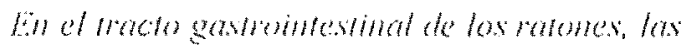

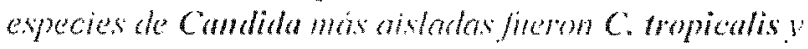

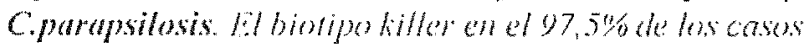

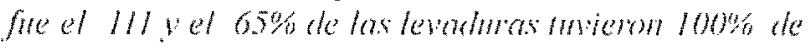
achividad killer.

\section{INTRODUCCION}

El cosistcma gastroincsimal hmmo es abicrto. integmolo. con midndes incractivis conchicndo mum chos habiat microbianos. micntras on ol amimal adulto normal. cada uno de cstos está coloniado por una comunidad microbiana aulóclona. Cormada por baclerias. protoroos y ocasionamente lovalunts. Cada ma de cstas ocupa su nicho conribuycho ca alguma mancra a ha cconomía de lodo cl cosistcmi.

El traclo gastrointestimal do los ratones sometidos at una dicta cquilibrada alberga bicterias y protoroos. no

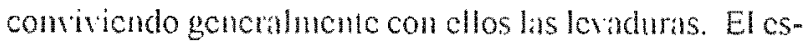
ado de cquilibrio de esc cosistema se ve alterido cuando cutran en juego ficlores diversos (dictas drogus. cle.) Si sus almontos son ricos en hidratos de carbono. desimrollam diversas especies de levadmas (1). Algums de cllas licnen la capacidad de achar sobre otras leradaras y microorganismos relacionados medinute a producción de

\section{SUMMARY}

The oblecties of this work were: ho study the kllow types af leasts wolated from the gastromestinat

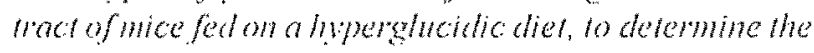

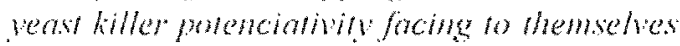

Foty Rockland adul mice were treated They were fal on a diet comprased of Graham flow (199), su-

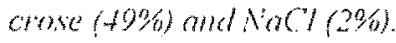

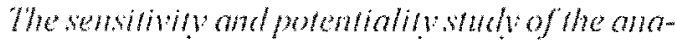
bed leasts when confirmed llah the lodian killer System (SKD) was performed according wo Polonelli et at.

In the gawtromberimal tract of mice, the species of Candith that shent he highes isolation were $C$. tropiculis ant Coparapsilosis. The most frequen hille upe was / I/ $07.5 \%$, and $65 \%$ of weasts presents $100 \%$ the killer achits.

sustancias lonles o towinas killor.

La producción por has levaduas de croloximas con actividad amimicrobiana, cs un fenómeno relatianculc comun en microorgmismos susceplibles y es mediada por recplores especificos de la pared cclukn $(2,3)$. Generalmenc corresponden a proteins g glicoprotemas. que son capaces de matar cólulas susceplibles pertenecientes a la misma especic o especies relacionadas y han sido delnidus cono loxinas killer. Las levaduras killer son inmunes a hacividad de sus propias loxinas killer y este efecto pucde representir un modelo de competición biológicá. que se manificsta de mancha similar a las bacleriocinas chirc las baclerias.

El crecto killer es mediado por la presencia de receptores colulares especíncos para his toximas y por la at!sencia de un sisicna de immundad especifica. Las loxinas killer son capices de malar levaluras no killer. asi como levdums de otros tipos killer. permancoiendo inmunes para su propin loxina "pan la producida por cepas del mismo 
grupo killer, pero son susceptibles a otms lovinas killer $(t)$.

El renotipo killer de las leviduris que se cipresa diforenciamone sobre cepas sensibles. ha sido utilizado en estudios cpidcmiológicos. como un mélodo licxible y seguro de biotipado de numerosis especies de microorm ganismos cucarionles y procarionles $(5)$.

Nuestro objelito, rue estudiar los tipos killer del traclo gastrointestinal de ratones somelidos a un dicla hiperglucida, detemimudo su potencialidud al cufrentarles culresi

\section{MATERIALES Y METODOS}

Se trataron 40 ratones Rockland adullos a los que se les suministró uma dicta compuesta de: harina de Graham $(49 \%)$. sicarosa $(49 \%)$ y $\mathrm{NaCl}(2 \%)$

Luego de 15 dias de trammicno con dien hiperglúcida. se sacrificó a los ralones. scccionindoles el intestino on cuatro partes: primera parte del incstino delgado (aproximadamente $10 \mathrm{~cm}$ ), segunda parte de intestino delgado $(15 \mathrm{~cm})$, cicgo y reclo.

Se cxtrajo h malcria fecal resuspendicndola on $\mid \mathrm{ml}$ de solución fisiológicn estéril. Sc ralizaron examenes direclos al fresco y con colonanc Gucguén para la obscriat ción de levaduras. Postcriormente se sembró cn medios de Sabouraud glucosa y Sabouraud glucosa t Car y se incubaron durance una scmana a $28^{\circ} \mathrm{C}$

Las levaduras fucron csludiadas por: producción de clamidoconidios cn agar harina de may, auxonograma de hidralos de carbono y sustancias nirogenadas. zimograma de hidatos de carbono. segin techica de Rippon y producción de ureasa on medio de Christensen (6).

Para la identificación de los géneros y cspecies de levaduras se utilizaron las claves de Kreger Van Rij (7)

La sensibilidad y potencialidad killer de las levadumas cafrenadas entre si, se basó en el Sistema Killer Ialiano (SKI). según la tćcnica de Polondli y col (8).

Se probó la scusibilidad de cakla una de lins levaduras con lodas las levaduras aishadas del traclo gastrointestinal de los mones.

\section{RESULTADOS Y DISCUSION}

Se aislaron 14 especies de levaduras desde las diferentes secciones del incto gastroinestinal de los ratones somelidos a dicta hiperglúcida; las especies de Candila más aisladas fucron $\mathrm{C}$. tropicalis $y \mathrm{C}$. parapsilosis. El biolipo killer, en cl $97.5 \%$ de los casos fue el 111. Una de las cepas de C. parapsilowis prescnló mu lipo killer 212 (Tabla. 1)

$\mathrm{E}(65 \%)$ de las levaduras aisladas luvicion $100 \%$ de aclividad killer. representadas mayoritariamente por 10 Tublu No!

Tipos killer de Cambida spp. aisladas ale tancto gastrointestinal de atones.

\begin{tabular}{|c|c|c|}
\hline Especic & No aislamicuto & Tivoskiller \\
\hline C. tronicalis & 12 & 111 \\
\hline C.porapsilowis & 10 & 111 \\
\hline C.az) & 3 & 111 \\
\hline C.multosa & 2 & 111 \\
\hline C.sulie & 2 & 111 \\
\hline C,quercitrasu & 2 & 111 \\
\hline C.buinensis & 1 & 111 \\
\hline C.cotenulata & 1 & 111 \\
\hline C.colliculosa & 1 & 111 \\
\hline C.famaha & 1 & 111 \\
\hline C.hrasici & 1 & 111 \\
\hline C.psembintern & & 111 \\
\hline C.fuerctum & 1 & 111 \\
\hline C.solant & 1 & 111 \\
\hline C.purapilossis & 1 & 212 \\
\hline
\end{tabular}

Tilbla No2.

Potencialidad libled de Cambidaspp. del tracto gastrointestinalde datones enfontadas cntresi.

\begin{tabular}{|c|c|c|c|}
\hline $\mathrm{N}^{0}$ aisham. & $\%$ & Especies & $\begin{array}{l}\text { nequidul } \\
\text { killen(\%) }\end{array}$ \\
\hline 26 & 65 & 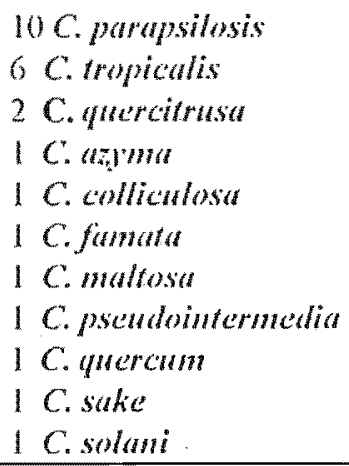 & 100 \\
\hline 3 & 7.5 & $\begin{array}{l}2 \text { C. atimua } \\
1 \text { C.knswi }\end{array}$ & 97 \\
\hline 3 & 7.5 & $\begin{array}{l}2 \text { C. tropicalis } \\
1 \text { C.purapsilosis }\end{array}$ & 4 \\
\hline 5 & 12,5 & $\begin{array}{l}\text { + C.tropicalis } \\
\text { I C.montosat }\end{array}$ & 9 \\
\hline $\begin{array}{l}1 \\
1 \\
1\end{array}$ & $\begin{array}{l}2.5 \\
2.5 \\
2.5\end{array}$ & $\begin{array}{l}\text { I C.bunimonsis } \\
\text { I C. catumuhata } \\
\text { I C. sake }\end{array}$ & $\begin{array}{l}86 \\
65\end{array}$ \\
\hline
\end{tabular}

cepas de C. parapsilasis y 6 de C. tropricalis, y un $15 \%$ con achividad corcana al $100 \%$ (Tabla,2). 
A pesar que existe cicra semejanza entre las principales especics de levaduras aisladas del tracto gastoinicstinal del ratóny los humanos (9), destacamos la ausencia de Colbicans, la cual representa el principal taxon on éstos úllimos (31\%) y su tipo killer más frecuentc $(67,1 \%)$, cl $111(9)$

El cfccto killer, ha sido ampliamente csludiado entre diferentes gencros y especics de lcvaduras, bacterias y olros microorganismos eucariontes $(10,11)$.

La mutalcza del fenómeno killer, implica un rol potencial para la competencia, considerando que las toxinas killer de levaduras pueden prevenir el acceso de microorganismos antagónicos a las fuentes. pudiendo conslituir una ventaja selectiva duranc las fases tempranas del crecimiento microbiano (t). Las toxinas killer de levaduras son producidas en formá óplima durme el crecimicno celuar y son activas contri células co d mismo estado cuando los muricntes cstán disponibles y cl pH es bajo.
La densidad de la población de levaduras susceptibles, creciendo en presencia de cepas killer, es mucho más baja que si la misma cepa susceptible de levadura crece en asociación con la cepa no killer (12). La probabilidad de que una toxina killer de levadura pueda matar a otras levaduras susceptibles, podría también depender de las características ecológicas, tales como la región, y el habitat del cual ambas ccpas de levaduras fueron colecladas (4). Esto podria explicar las diferencias encontradas en el tracto gastrointestinal del modelo animal. Por otra parte, en el tracto gastrointestinal de los ratones, observamos que la mayoría de las levaduras, tienen ol $100 \%$ de, actividad killer, lo que indicaría un mayor poder palógeno, tal como lo describió Morace et al., (13). Asimismo, no habria variación de cepas dentro de las especies, excepto para una copa de C.parapsilosis.

\section{REFERENCIAS}

1.- Magaro, IIM, a Bracalemi, B.,.C.ate (1985). Introlacioness entre protozoarios y levalmas intestinales de mon. Bolotin Chileno de Pufasiologia. $40: 7-13$

2.- Polanelli, L. \& Morate, G. (1986). Reowaluation of the yeast killer plenemenom, I.Clin. Nicrobiol. 24:860-869)

3.- Polmelli, L; Conti, S.; Gerimu, M.; Maghmi, W, Norace, G.; Cheza, $\mathrm{C}$ (1991) interfiess of the yeas killer phenomenon. Crit. Revilicrobinl 18:47-87

4, Maglian, W; Conti, S.; Gerioni, M.; Bertoloti, D. and polonnil, L.(1997). Yenst killer system. Clin. Microbiol. Rev.10:369400

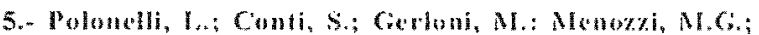

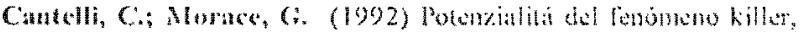
dei liciti. Rer. Harom. Mlicol. 9:23-27

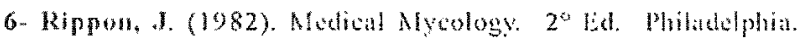
USA. Situnders Company, pp. 774-775

7.- Kreger- Van Rij, N.J.W. (1984), The yeas. A Thxomomy study: $3^{\circ}$ Ea. Ekerfer Sciente Publisher B.V. Ansterdam.
B.-Polonkh, L.; Arehibusaci, $C$; Sestito, M.; Norace, $G$. (1983). Killer System; A simple method for dilerentiang Cantida abicans strains. J.Clin. Microbiol. 17:774-780

9.- Magaro, IIAL; Basoli, M.S.; Thomas, C.E.; Eehenique, C.G.; Tosello, M.K.; Mraculenti, B.J.C. de.(1993). Aspestos del ecosistema gastrointestinil humano y levadiuas killer. Rev. lberoam. Micol. 10:47-50

10.- Polbnell, L.\& Morace, J. (1986). Reevaluation of the Yeasts hiller Phenomenon. J. Clin. Niciobiol. 24:866-869

11 - Prosest, H; lolonelli, L.; Cont, S; lisicaro, P; Geriont, M. ; Hoiron, P. (1925). Use of Vean killer System to idemify species of the Necardia asteroules complex. J. Clin.Mlerobiol. $33: 8-10$

12- Gunten, PI \& Stamer, WT. (1292). Killer fuctor as a medianism of interference competition in yeast associated with andi. Wology. 73:54-67

13.- Mutace, G, Mrehibusace, C.; Sestien, M.; Poloncll, L.(1984). Stain dileremiation of pathogenic yeasts by the killer system. Mycopathol, 84:81.85 